\title{
Health-Related Genetic Direct-to-Consumer Tests in the German Setting: The Available Offer and the Potential Implications for a Solidarily Financed Health-Care System
}

\author{
Marika Plöthner Mike Klora Daniel Rudolph \\ Johann-Matthias Graf von der Schulenburg \\ Center for Health Economics Research Hannover (CHERH), Leibniz University Hannover, Hannover, Germany
}

\section{Keywords}

Direct-to-consumer genetic testing $\cdot$ Health-related offer .

Germany · Health-care system

\begin{abstract}
Background: The global genetic direct-to-consumer (DTC) market will reach a volume of USD 230 billion in 2018. The expenditures for this genetic analysis are borne by the customer, whereas consequential costs may arise for a solidarily financed system. In a first step, it is essential to gain an overview of the currently available offer in the German setting. Methods: In April 2016, we conducted a systematic internet search in the Google search engine. In November 2016, we updated the information of the webpages in terms of country, language, types of health-related tests, additional offer of non-health-related DTC test, information about sensitivity and specificity, certification and accreditation, costs as well as reference to German Act on Genetic Testing. Results: Thirty-five webpages were included in the final overview. A plurality of different predictive analysis options was identified. Price information was not available for all offered genetic analyses. Costs for predictive analysis in one disease vary between EUR 90 and 990, for predictive package analysis between EUR 232.18 and 375, and for genetic lifestyle analysis
\end{abstract}

\section{KARGER}

E-Mail karger@karger.com

www.karger.com/phg between EUR 84.55 and 570.20. Conclusions: Genetic results may lead to uncertainty and anxiety; therefore, subsequent costs for a solidarily financed system may arise. Genetic DTC tests may have an influence on different players on the micro-, meso- and macro-levels, which may have a cost-cutting or cost-increasing effect on health-care expenditures. The increased interest in genetic analysis as well as the possibility of worldwide internet-based access to genetic tests requires population-wide education.

(c) 2017 S. Karger AG, Basel

\section{Introduction}

The community is characterized by an increased health consciousness [1]. Patients are becoming increasingly responsible and informed and also want to take a proactive role in their health-care management [2]. Besides the traditional health-care sector (e.g., medical services by physicians), citizens are using additional sources for getting health information [3]. For this purpose, the internet constitutes an essential tool for health information access [4]. Health information is thus easily available from home and the-so called "Dr Google" is often the primary source for health-related questions [5]. 
In the genomic century, the knowledge of health-influencing genetic factors is steadily increasing [6]. The two main factors for the growing genetic knowledge are: First, progress in genetic technology, which is characterized by cheaper and faster genetic analysis [7]; and second, increased genomic knowledge as a result of genome wide-association studies (GWAS). GWAS enable a better understanding of genotype and phenotype correlations and also provide information about the probability for the development of specific diseases [8-10]. Hence, genetic information can be used for diagnosis, prognosis, and treatment [11] as well as for a "genome-guided preventive medicine" [12]. Based on the known associations between genetics and common diseases, the demand for genetic analysis has increased, particularly with a preventive approach [13].

In Germany, preventive or predictive genetic analyses without a direct and specific indication or only for interested people are basically not part of the standard benefit of the statutory health insurance. As a result, such genetic analyses can be accessed through case-by-case decision (e.g., predictive analysis for the risk of breast cancer due to the family history) or private payment. Besides the traditional market, an internet-based market - the so called direct-to-consumer (DTC) market - has been developed. The development of this market has been encouraged by a lower price, no geographic restriction, as well as the easy availability from home $[14,15]$. Estimates suggest that the global genetic DTC market will reach a volume of USD 230 billion in 2018 [16]. DTC genetic tests are directly addressed to customers via television, print advertisements, or the internet [17]. Customers receive a test kit and send a sample (mostly saliva) back to the company. After analyzing the genetic material in their own or an outsourced laboratory, the information about the personal genetic makeup will subsequently be sent to the customers [18].

Overall, genetic DTC tests can be differentiated into health-related and non-health-related tests. Tests for identity (e.g., paternity and ancestry) can be classified as non-health-related DTC tests, whereas diagnostic and predictive tests (risk of disease) as well as lifestyle tests (nutrition, aging, behavior, etc.) can be classified as health-related tests [19-21]. In contrast to predictive or diagnostic genetic tests, lifestyle tests can be attributed a lower clinical value [21]. Therefore, the German Act on Genetic Testing (GenDG) heavily regulates the execution of genetic analysis with a predictive or diagnostic approach, which makes the integration of a physician necessary. However, this law is only obligated for companies located in Germany, and the access to offers of companies located in other countries is not restricted. According to the increased demand for genetic analysis [22] and the associated increasing use of predictive and therapeutic measures, it is important to analyze which kinds of genetic analysis the population is confronted with. In general, the costs for the execution of a genetic DTC test are borne by the customers, whereas follow-up costs may arise in the solidarily financed system. An informed patient develops other priorities, and this may have an influence on the different players in the health-care system. For this purpose, we conducted a systematic internet analysis to identify the currently available health-related genetic DTC offers in the German setting. Therefore, the review had the following objectives: (1) to identify the offer of health-related genetic DTC tests in the German setting; (2) to highlight and discuss the implications of genetic DTC tests for a solidarily financed health-care system

\section{Methods}

First, review elements (objective/aim, inclusion and exclusion criteria, and outcomes) were defined in order to focus on the scientific issue and facilitate the internet search (Table 1).

In April 2016, we conducted a systematic internet search using the Google search engine. To generate the maximum number of heterogeneous hits, we deactivated the installed adblocker as well as the standard activated web protocol. For the search strategy, we used single and combined terms in the German language. For single terms, we used different common German terms for genetic analysis and combined the most common with genetic DTC testspecific terms. Accordingly, the following search strategies were used: (1) genetic analysis, (2) genetic test, (3) genetics test, (4) genetic testing, (5) DNS testing, (6) DNA analysis, (7) genetics analysis, (8) DNA testing, (9) DNS analysis, (10) genetic analysis, (11) DNS test, (12) DNA test, (13) genetic test, (14) genetic analysis AND diseases, (15) genetic analysis AND lifestyle, (16) genetic analysis AND health, (17) genetic analysis AND private, (18) genetic analysis AND at home, (19) genetic analysis AND cancer, (20) DNS test AND diseases, (21) DNS test AND lifestyle, (22) DNS test AND health, (23) DNS test AND private, (24) DNS test AND at home, (25) DNS test AND cancer, (26) DNA test AND diseases, (27) DNA test AND lifestyle, (28) DNA test AND health, (29) DNA test AND private, (30) DNA test AND at home, (31) DNS test AND cancer, (32) genetic test AND diseases, (33) genetic test AND lifestyle, (34) genetic test AND health, (35) genetic test AND private, (36) genetic test AND at home, (37) genetic test AND cancer.

The systematic internet search was performed independently on two computers. Furthermore, the assessment of the webpages was also conducted independently by 2 researchers. Disagreements were resolved through discussion. Criteria for inclusion were as follows: (1) webpages offering a health-related genetic DTC test (diagnostic and predictive tests as well as lifestyle-tests); (2) tests were primarily targeted at customers (not physicians or 
Table 1. Review objective

\begin{tabular}{ll}
\hline Objective/aim & $\begin{array}{l}\text { To point out the available offer of health-related genetic DTC tests; highlight the } \\
\text { potential implications of health-related genetic DTC tests for a solidarily financed } \\
\text { health-care system }\end{array}$ \\
\hline Inclusion criteria & $\begin{array}{l}\text { Webpages that offer health-related genetic DTC tests (predictive and diagnostic } \\
\text { tests as well as lifestyle tests; webpages were not restricted to specific predictive/ } \\
\text { diagnostic analysis or lifestyle tests or registered offices and languages; tests were } \\
\text { targeted at customers; direct selling webpages }\end{array}$ \\
\hline Outcomes & $\begin{array}{l}\text { Type of tests and costs; further comparative aspects (registered offices, languages, } \\
\text { additional supplement of non-health-related tests, information about sensitivity } \\
\text { and specificity, reference to GenDG and information about certification and/or } \\
\text { accreditation) }\end{array}$ \\
\hline
\end{tabular}

other medical providers); and (3) direct selling webpages (webpages only with test links or advertisements were not included) (see Table 1). In the course of an update in November 2016, offers and the provided information in the webpages were actualized. The included webpages were not restricted to countries, languages, and specific types of genetic analysis. To ensure comparability, the costs were converted to EUR at the exchange rate of the current year [23].

\section{Results}

The systematic internet search retrieved 11,199 webpages. After screening for eligibility, 35 webpages were included in the final overview (Fig. 1).

The webpages are characterized by a variety of aspects, such as country, language, types of health-related tests, cost of the specific tests, information about sensitivity and specificity, information about certification and accreditation, as well as information about the availability of and additional supplement of non-health-related tests. A detailed overview is provided in Table 1.

\section{Country and Language}

The majority of included webpages $(17 \times)$ have their headquarters located in Germany, followed by Switzerland $(3 \times)$ and Slovenia $(3 \times)$. Overall, 12 different countries were identified as company headquarters. Twelve of the included webpages present their offer only in German, whereas 8 of these are German companies and 4 are foreign companies. One company provides a translation service, whereby a variety of languages is available. Furthermore, one company provides a direct selection of 11 languages. Five of the included webpages provide no German translation, whereas 4 of these are only represented in English.

Health-Related Genetic DTC Tests in the German Setting

\section{Offer of Health-Related DTC Tests}

Eleven webpages offer only genetic lifestyle tests and 16 webpages offer only predictive or diagnostic genetic DTC tests. An offer of genetic lifestyle DTC tests as well as predictive and diagnostic genetic DTC was identified on 8 webpages. Of the 17 webpages with their headquarters located in Germany, 5 offer genetic lifestyle tests, 8 predictive and diagnostic DTC tests, and 4 both types of genetic analysis. The offer of the 8 webpages represents one genetic analysis in the diagnostic content (lactose intolerance gene mutation analysis) and 7 genetic analyses in the predictive content (prenatal test [3×], tests of therapeutic safety and tests of genetic disease dispositions $[3 \times])$.

Generally, in terms of predictive analysis, a plurality of genetic analyses were identified for general diseases (type 1 and 2 diabetes, migraine etc.), immune system (lupus, multiple sclerosis etc.), aging (Alzheimer disease, osteoporosis, etc.), cancer dispositions (bladder, breast, prostate, colon, etc.), and pharmacogenetic testing (antidepressants, oncology products, etc.). Thereby, customers have the possibility to choose between predictive analyses especially for single diseases $(8 \times)$ or purchase a package with an analysis of different genetic dispositions $(9 \times)$. Four providers offer a mixed range of single and/or package analysis. For example, in terms of prenatal analysis, some offers provide a choice between a single analysis of trisomy 21 or trisomy 21,18 , and 13; additionally with sex determination, chromosomal abnormality, and/or sex chromosomal disorders. Some providers offer a package of genetic analyses for up to 35 diseases, gender-specific analysis, and an additional analysis of pharmacogenetics effects (partly up to 230 drugs). Genetic lifestyle DTC tests for weight, nutrition, and fitness are represented by a variety of different

Public Health Genomics 2017;20:203-217 205 


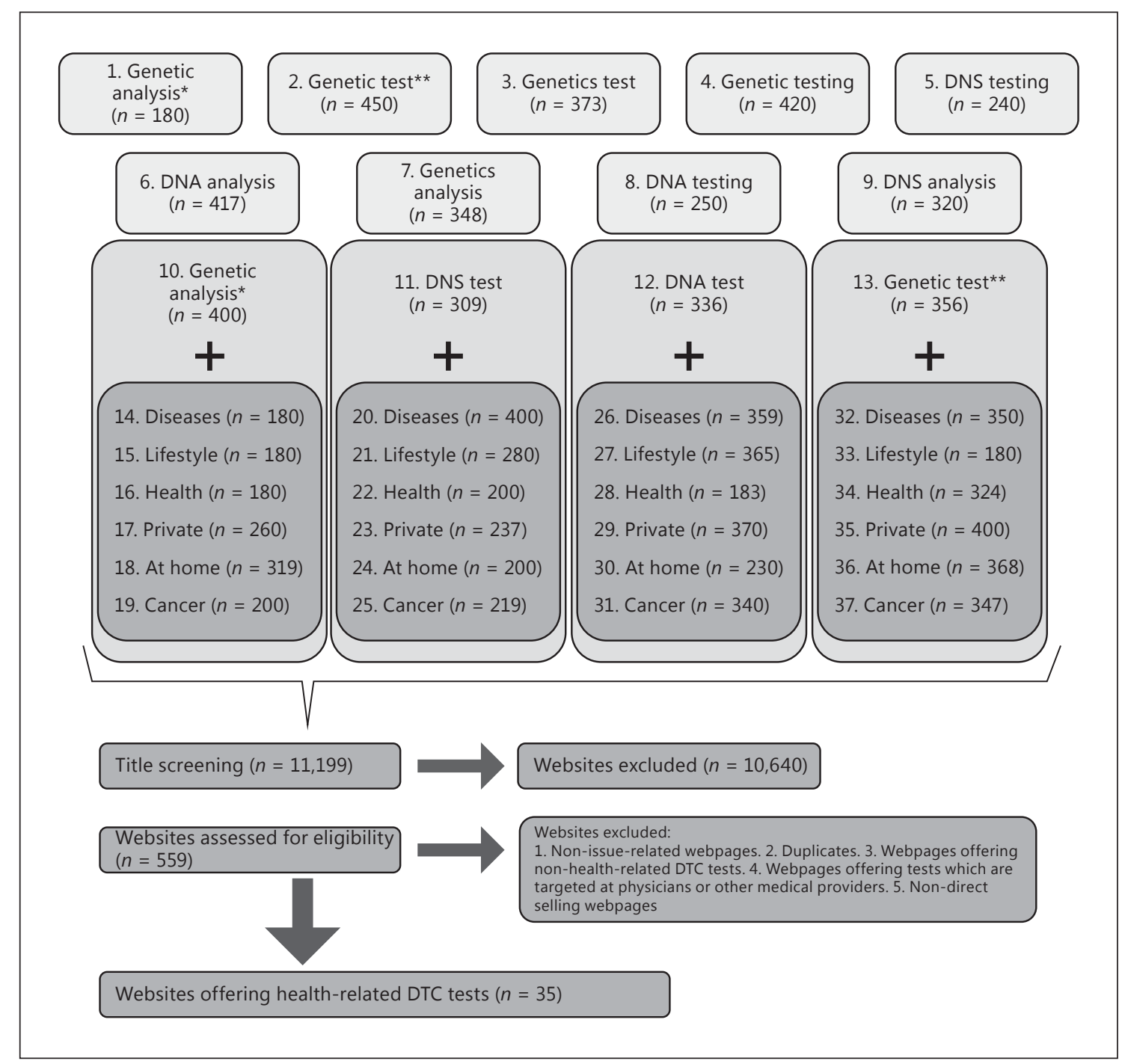

Fig. 1. Flow diagram of websites identified on the basis of inclusion criteria. ${ }^{*}{ }^{* *}$, two different search terms in the German language; $n$, number of displayed hits.

terms (e.g., gene-weight analysis, metabolic analysis, nutrigenetic analysis, fitness). Some bundled offers provide an analysis in areas of weight, nutrition, and fitness, and others focus only on one area. Moreover, genetic analysis for hair loss, body height, day or night person, memory performance, etc. was also identified. Furthermore, the majority of the included providers focus only on health-related analysis. Only 6 webpages provide an additional supplement of non-health-related genetic DTC test (paternity test, test for relationship etc.).
Costs of Predictive and Diagnostic Genetic DTC Tests

Most of the companies $(20 \times)$ provide price information for all offered analyses. However, the webpages of 10 companies do not communicate any price information. Three companies provide price information only for a few analyses. Two webpages directly refer price information on request online or by physician or hotline. There is a wide variety of prices. The cost for predictive single analysis can vary between EUR 89 (e.g., test for risk of thrombosis) and EUR 990 (e.g., test for risk of dystrophy or test for risk of metabolic disease). The cost for predictive package analysis varies between EUR 232.18 (test of

Plöthner/Klora/Rudolph/ Graf von der Schulenburg 
risk for 34 different diseases) and EUR 375 (test of risk for 28 common diseases). The identified prices for pharmacogenetic testing range between EUR 160.88 (test for therapeutic safety of clopidogrel) and EUR 395.49 (test for therapeutic safety of antidepressants). Cost differences of EUR 220 and 395.49 for an efficacy test of tamoxifen were identified. In the diagnostic content, especially in terms of genetic analysis for dietary intolerances, costs between EUR 69 (e.g., lactose) and EUR 700 (e.g., food intolerances) were found. Prices for prenatal testing vary according to the extent of genetic analysis (e.g., trisomy 21 or trisomy $21,18,13$ ) and partly on the processing time. Hence, prices are between EUR 249 and 649.42.

\section{Costs of Lifestyle-Related Testing}

The cost for genetic lifestyle analysis varies between EUR 84.55 (analysis for obesity and overweight) and EUR 570.20 (genetic weight analysis).

\section{Additional Information}

Six webpages provide information about sensitivity and/or specificity. Five of these are located in Germany and one in Canada. Another provider advertises regarding "highest accuracy" [32]. All of these are offers in a predictive or diagnostic context. Of all 35 webpages included, 10 provide some information about the GenDG. All of these, except one, are located in Germany. All except 14 provide information about certification and/or accreditation. The majority of these are located in Germany.

\section{Discussion}

This comprehensive review presents an overview about the current available health-related genetic DTC tests in the German setting. It shows that a variety of health-related but also non-health-related DTC tests exist. The offer of health-related genetic DTC tests is represented by lifestyle tests as well as predictive and diagnostic tests. According to the GenDG, predictive and diagnostic genetic tests are restricted to physicians, whereas predictive tests may only be performed by human geneticists. However, some companies created possibilities to offer genetic tests while meeting the conditions of the GenDG. Examples of these identified methods are as follows. (1) The execution of the genetic analysis (laboratory process) will only be performed after a genetic counselling and with the signature of a qualified physician [31], or the genetic test can only be ordered by or with a

Health-Related Genetic DTC Tests in the German Setting physician, and afterwards the results are transmitted to the physician. (2) Companies additionally provide the possibility of genetic counselling by their own physician [35]. Therefore, the properties of a genetic DTC test in a strict sense (order, saliva sample, and receipt of results at home) are not fulfilled by such access methods. However, the offer is directly addressed to the customer, and the additional integration of physicians for German-located companies could be a possibility to conform to the GenDG.

The GenDG is an act that highly restricts access to genetic analysis. However, in Europe there are no common regulations for genetic analyses, and therefore also not for DTC tests. Borry et al. [59] provide an overview about the different regulations for genetic analyses in 7 European countries (Belgium, Germany, France, the Netherlands, Portugal, Switzerland, and UK). This report shows that none of these countries has specific regulations for genetic DTC testing. Rather, it is a transfer of the existing legislation to the DTC context. In some of these countries (France, Germany, the Netherlands, Portugal, and Switzerland), genetic analyses are restricted to physicians, and genetic counselling is obligatory. Due to the fact of missing European regulations and the unlimited access to various international genetic DTC offers, some organizations and agencies (for example, Initiative of European Academies Science Advisory Council [EASAC] and the Federation of European Academies of Medicine [FEAM] [60] or the Human Genetics Commission [61]) published statements for information and education about DTC genetic testing.

This market analysis provides an overview of the existing variety of health-related genetic testing (genetic predictive or diagnostic tests as well as lifestyle analysis) in the German setting. The supplement is characterized by a plurality of aspects, which makes a comparison for customers difficult. In the context of lifestyle analysis, interested people are faced with different terms, extensions of analysis as well as prices. In the predictive context, people have to decide between analysis for one specific disease and a package analysis. Costs for single analysis are partly more expensive than those for comprehensive analysis. Moreover, information about the amount of investigated gene variations may be difficult for costumers to evaluate [62]. Furthermore, tests and costs for a specific indication, for example the risk of thrombosis (Table 2) are not or hardly comparable for customers. Test cost varies with the analysis of the specific targets. However, mostly there is no information about it, and this shows a lack of transparency for interested people.

Public Health Genomics 2017;20:203-217 207 
Table 2. Overview of included webpages

\begin{tabular}{|c|c|c|c|c|c|c|c|c|}
\hline \multicolumn{3}{|l|}{ General information } & \multicolumn{3}{|l|}{ Offer } & \multicolumn{3}{|c|}{ Additional information } \\
\hline company & $\begin{array}{l}\text { registered } \\
\text { office }\end{array}$ & languages & health-related tests & costs & $\begin{array}{l}\text { non- } \\
\text { health- } \\
\text { related } \\
\text { tests }\end{array}$ & $\begin{array}{l}\text { sensitivity or } \\
\text { specificity/ } \\
\text { accuracy }\end{array}$ & $\begin{array}{l}\text { reference } \\
\text { to GenDG }\end{array}$ & $\begin{array}{l}\text { certification } \\
\text { and/or } \\
\text { accreditation }\end{array}$ \\
\hline $\begin{array}{l}\text { Abnehmgene } \\
{[24]}\end{array}$ & Spain & German & Gene-weight analysis & $\begin{array}{l}\text { EUR } \\
398 / 419^{1}\end{array}$ & No & ns & ns & Yes \\
\hline $\begin{array}{l}\text { Adison } \mathrm{Lab}^{2} \\
{[25]}\end{array}$ & Ireland & $\begin{array}{l}\text { Spanish, } \\
\text { English, } \\
\text { French, } \\
\text { German, } \\
\text { Italian, } \\
\text { Russian, } \\
\text { Romanian }\end{array}$ & Genetic analyses of food intolerances & EUR 700 & No & ns & ns & ns \\
\hline Amway [26] & Germany & $\begin{array}{l}\text { German, } \\
\text { English, } \\
\text { Turkish, } \\
\text { Russian }\end{array}$ & Weight & EUR 570.20 & No & ns & ns & ns \\
\hline \multirow[t]{3}{*}{ Ceneta [27] } & \multirow[t]{3}{*}{ Germany } & $\begin{array}{l}\text { German, } \\
\text { English, } \\
\text { Turkish }\end{array}$ & $\begin{array}{l}\text { Trisomy } 21,18,13 \text {, and sex chromosomal } \\
\text { disorders and XY chromosomal disorder } \\
\text { (as required for sex determination) }\end{array}$ & EUR 299 & \multirow[t]{3}{*}{ No } & \multirow[t]{3}{*}{ Yes } & \multirow[t]{3}{*}{ Yes } & \multirow[t]{3}{*}{ ns } \\
\hline & & & $\begin{array}{l}\text { Trisomy } 21,18,13 \text { (as required sex } \\
\text { determination) }\end{array}$ & EUR 299 & & & & \\
\hline & & & Trisomy 21 (as required sex determination) & EUR 249 & & & & \\
\hline Coloalert [28] & Germany & German & Colon cancer prevention test & EUR 98.50 & No & Yes & ns & Yes \\
\hline \multirow[t]{4}{*}{ DNAdirekt [29] } & The & German & Risk of thrombosis & EUR 99 & Yes & ns & ns & Yes \\
\hline & \multirow{3}{*}{\multicolumn{2}{|c|}{ Netherlands }} & Risk of hemochromatosis & EUR 99 & & & & \\
\hline & & & Prader-Willi syndrome & ns & & & & \\
\hline & & & Apo-E Alzheimer disease & EUR 99 & & & & \\
\hline \multirow[t]{3}{*}{ DNAFit [30] } & \multirow[t]{3}{*}{ UK } & \multirow[t]{3}{*}{ English } & $\begin{array}{l}\text { Diet (diet type, carbohydrates response, saturated } \\
\text { fat response, lactose intolerance) (different } \\
\text { packages: core info or more than core info or all } \\
\text { information) }\end{array}$ & $\begin{array}{l}\text { EUR 119- } \\
239\end{array}$ & \multirow[t]{3}{*}{ No } & \multirow[t]{3}{*}{ ns } & \multirow[t]{3}{*}{ ns } & \multirow[t]{3}{*}{ ns } \\
\hline & & & $\begin{array}{l}\text { Fitness (different packages: core info: power } \\
\text { endurance response, sports injury resilience, } \\
\text { recovery speed, recovery nutrition needs) or all } \\
\text { information (aerobic }\left[\mathrm{VO}_{2} \mathrm{Max}\right] \text { potential, fitness } \\
\text { genotype breakdown) }\end{array}$ & $\begin{array}{l}\text { EUR 145- } \\
179\end{array}$ & & & & \\
\hline & & & Diet and fitness & EUR 338 & & & & \\
\hline $\begin{array}{l}\text { DNA Plus- } \\
\text { Zentrum für } \\
\text { Humangenetik } \\
{[31]}\end{array}$ & Germany & $\begin{array}{l}\text { German, } \\
\text { English } \\
\text { (UK and } \\
\text { US), } \\
\text { Hungarian }\end{array}$ & $\begin{array}{l}\text { Nutrition (dietary, weight); digestion (lactose, } \\
\text { gluten, colon); metabolism (iron, diabetes, } \\
\text { Alzheimer, detoxification); movement (sport, } \\
\text { joints, bones); pharma (drugs, remuneration, } \\
\text { hormone replacement); eyes (glaucoma, macula); } \\
\text { heart (thrombosis, heartbeat, blood pressure, } \\
\text { artery); beauty (hair loss male and female, } \\
\text { periodontitis); trainees (pregnancy, babyl00+); } \\
\text { cancer (prostate, breast); allergy }\end{array}$ & ns & No & ns & Yes & ns \\
\hline \multirow{10}{*}{$\begin{array}{l}\text { DTC } \\
\text { DNATesting } \\
\text { Center [32] }\end{array}$} & \multirow[t]{10}{*}{ Austria } & \multirow[t]{10}{*}{ German } & Risk of heart attack & EUR 890 & \multirow[t]{10}{*}{ Yes } & \multirow[t]{10}{*}{$\mathrm{ns}^{3}$} & \multirow[t]{10}{*}{$\mathrm{Yes}^{4}$} & \multirow[t]{10}{*}{ Yes } \\
\hline & & & Risk of cancer & EUR 790 & & & & \\
\hline & & & Risk of diabetes & EUR 290 & & & & \\
\hline & & & Risk of stroke & EUR 640 & & & & \\
\hline & & & Risk of osteoporosis & EUR 490 & & & & \\
\hline & & & Point of aging & EUR 990 & & & & \\
\hline & & & Risk of cystic fibrosis & EUR 890 & & & & \\
\hline & & & Risk of anemia & EUR 790 & & & & \\
\hline & & & Risk of dystrophy & EUR 990 & & & & \\
\hline & & & Risk of metabolic disease & EUR 990 & & & & \\
\hline
\end{tabular}


Table 2 (continued)

\begin{tabular}{|c|c|c|c|c|c|c|c|c|}
\hline \multicolumn{3}{|c|}{ General information } & \multicolumn{3}{|l|}{ Offer } & \multicolumn{3}{|c|}{ Additional information } \\
\hline company & $\begin{array}{l}\text { registered } \\
\text { office }\end{array}$ & languages & health-related tests & costs & $\begin{array}{l}\text { non- } \\
\text { health- } \\
\text { related } \\
\text { tests }\end{array}$ & $\begin{array}{l}\text { sensitivity or } \\
\text { specificity/ } \\
\text { accuracy }\end{array}$ & $\begin{array}{l}\text { reference } \\
\text { to GenDG }\end{array}$ & $\begin{array}{l}\text { certification } \\
\text { and/or } \\
\text { accreditation }\end{array}$ \\
\hline \multirow{3}{*}{$\begin{array}{l}\text { DNAnutri } \\
\text { Control [33] }\end{array}$} & \multirow[t]{3}{*}{ Austria } & \multirow{3}{*}{$\begin{array}{l}\text { English, } \\
\text { German }\end{array}$} & Weight sensor & ns & \multirow[t]{3}{*}{ No } & \multirow[t]{3}{*}{ ns } & \multirow[t]{3}{*}{ Yes } & \multirow[t]{3}{*}{ Yes } \\
\hline & & & Nutrition sensor & ns & & & & \\
\hline & & & Sport sensor & ns & & & & \\
\hline $\begin{array}{l}\text { DNA Weight } \\
\text { Control [34] }\end{array}$ & \multicolumn{2}{|c|}{$\begin{array}{c}\text { Switzerland German, } \\
\text { English }\end{array}$} & Genetic metabolic analysis & ns & No & ns & ns & ns \\
\hline $\begin{array}{l}\text { Dr. Seibt } \\
\text { Genomics [35] }\end{array}$ & Germany & $\begin{array}{l}\text { German, } \\
\text { English }\end{array}$ & $\begin{array}{l}\text { Several tests for: DNA and diseases (alpha-1- } \\
\text { antitrypsin-deficiency, Alzheimer, atherosclerosis } \\
\text { risk, caffeine, Gilbert syndrome, hereditary } \\
\text { hemochromatosis, histamine intolerance, } \\
\text { hyperlipoproteinemia, lactose intolerance, } \\
\text { Bekhterev's disease, Crohn's disease, periodontitis } \\
\text { risk, risk of thrombosis (factor 2, factor 5, PAI-1, } \\
\text { MTHFR), celiac disease; panel-analyses: adiposity, } \\
\text { Alzheimer disease/dementia, BRCA1\&2, } \\
\text { BRCA1\&2 ovarian carcinoma, cardiologic } \\
\text { analysis, dermatologic analysis, metabolism of } \\
\text { drugs and exogenous substances, type } 2 \text { diabetes, } \\
\text { hereditary disease, estrogen-specific analysis, } \\
\text { glaucoma, health, capsule fibrosis, cancer hotspot, } \\
\text { macula, osteoporosis, pharmacogenomics, } \\
\text { prostate, starter); DSG-combi: three different, } \\
\text { DSG sequencing: WES, WGS } \\
\text { Liquid biopsy marker: } 23 \text { different; liquid biopsy } \\
\text { panel and liquid biopsy exome } \\
\text { Pharmacogenetics tests: antidepressants (13 } \\
\text { different); atypical neuroleptics, beta-blockers, } \\
\text { oncological (9 different), opiates, proton pump } \\
\text { inhibitor (2 different), statins, thrombocyte } \\
\text { aggregation inhibitors; DSG-combi analysis (5 } \\
\text { different); drug interaction check }\end{array}$ & $\begin{array}{l}\text { price on } \\
\text { request }\end{array}$ & No & ns & Yes & ns \\
\hline EasyDNA [36] & Switzerlanc & $\begin{array}{l}\text { German, } \\
\text { English, } \\
\text { French }\end{array}$ & $\begin{array}{l}\text { Analysis includes: cancer predisposition (bladder, } \\
\text { breast, colon, stomach, lung, prostate, skin), } \\
\text { general (overweight, migraine, type } 1 \text { and } 2 \\
\text { diabetes), aging (Alzheimer disease, osteoarthritis, } \\
\text { rheumatoid arthritis), cardiovascular events } \\
\text { (aneurysm, atrial fibrillation, venous } \\
\text { thromboembolism, peripheral arterial occlusive } \\
\text { disease, heart diseases); immune system (lupus, } \\
\text { Graves' disease, celiac disease, multiple sclerosis, } \\
\text { psoriasis) }\end{array}$ & $\begin{array}{l}\text { CHF 375 } \\
(\text { EUR } \\
347.01)\end{array}$ & No & ns & ns & Yes \\
\hline $\begin{array}{l}\text { FutureGenetic } \\
{[37]}\end{array}$ & Canada & $\begin{array}{l}\text { German, } \\
\text { English }\end{array}$ & $\begin{array}{l}\text { Test includes } 28 \text { of the most common diseases: } \\
\text { age-related macular degeneration, alopecia, } \\
\text { Alzheimer disease, atrial fibrillation, basal cell } \\
\text { carcinoma, bladder cancer, breast cancer, celiac } \\
\text { disease, colon cancer, coronary heart disease, } \\
\text { exfoliations glaucoma, stomach cancer, Graves' } \\
\text { disease, intracerebral aneurysm, lung cancer, } \\
\text { lupus, melanoma, migraine, multiple sclerosis, } \\
\text { obesity, open-angle glaucoma, peripheral arterial } \\
\text { occlusive disease, prostate cancer, psoriasis, } \\
\text { rheumatoid arthritis, type } 1 \text { and } 2 \text { diabetes, venous } \\
\text { thromboembolism }\end{array}$ & EUR 375 & No & ns & ns & Yes \\
\hline
\end{tabular}


Table 2 (continued)

\begin{tabular}{|c|c|c|c|c|c|c|c|c|}
\hline \multicolumn{3}{|l|}{ General information } & \multicolumn{3}{|l|}{ Offer } & \multicolumn{3}{|c|}{ Additional information } \\
\hline company & $\begin{array}{l}\text { registered } \\
\text { office }\end{array}$ & languages & health-related tests & costs & $\begin{array}{l}\text { non- } \\
\text { health- } \\
\text { related } \\
\text { tests }\end{array}$ & $\begin{array}{l}\text { sensitivity or } \\
\text { specificity/ } \\
\text { accuracy }\end{array}$ & $\begin{array}{l}\text { reference } \\
\text { to GenDG }\end{array}$ & $\begin{array}{l}\text { certification } \\
\text { and/or } \\
\text { accreditation }\end{array}$ \\
\hline \multirow[t]{8}{*}{$\begin{array}{l}\text { Genetic balance } \\
{[38]}\end{array}$} & \multirow[t]{8}{*}{ Germany } & \multirow[t]{8}{*}{ German } & $\begin{array}{l}\text { Genetic balance (diet, weight, nutrition) basic } \\
\text { package }^{5}\end{array}$ & EUR 399.00 & \multirow[t]{8}{*}{ No } & \multirow[t]{8}{*}{ ns } & \multirow[t]{8}{*}{ Yes } & \multirow[t]{8}{*}{ Yes } \\
\hline & & & $\begin{array}{l}\text { Genetic balance (diet, weight, nutrition) medium } \\
\text { package }^{5}\end{array}$ & EUR 419.00 & & & & \\
\hline & & & $\begin{array}{l}\text { Genetic balance (diet, weight, nutrition) premium } \\
\text { package }^{5}\end{array}$ & EUR 449 & & & & \\
\hline & & & Health control - cardiovascular diseases & ns & & & & \\
\hline & & & Health control - metabolism & ns & & & & \\
\hline & & & Health control - bone health & ns & & & & \\
\hline & & & Health control - eye health & ns & & & & \\
\hline & & & $\begin{array}{l}\text { Health control - Alzheimer disease and } \\
\text { prevention }\end{array}$ & ns & & & & \\
\hline \multirow[t]{9}{*}{ Genoris [39] } & \multirow[t]{9}{*}{ Italy } & \multirow{9}{*}{$\begin{array}{l}\text { Italian, } \\
\text { German }\end{array}$} & DNA and weight & ns & \multirow[t]{9}{*}{ No } & \multirow[t]{9}{*}{ ns } & \multirow[t]{9}{*}{ ns } & \multirow[t]{9}{*}{ Yes } \\
\hline & & & DNA and nutrition & ns & & & & \\
\hline & & & $\begin{array}{l}\text { Premium plus sensor female/male: } 35 \text { different } \\
\text { diseases/ } 110 \text { gene variations/effect or adverse } \\
\text { events of } 230 \text { drugs }\end{array}$ & ns & & & & \\
\hline & & & $\begin{array}{l}\text { Premium sensor female/male: } 22 \text { different } \\
\text { diseases/90 gene variations/ adverse events of } 230 \\
\text { drugs }\end{array}$ & ns & & & & \\
\hline & & & $\begin{array}{l}\text { Female sensor: } 12 \text { different diseases/65 gene } \\
\text { variations/ adverse events of } 73 \text { drugs/assessment } \\
\text { of risks and advantages of hormone replacement } \\
\text { therapy (especially for women } 40+\text { ) }\end{array}$ & ns & & & & \\
\hline & & & $\begin{array}{l}\text { Male sensor: } 65 \text { gene variations, } 12+\text { different } \\
\text { diseases, adverse events of } 73 \text { drugs, assessment } \\
\text { of risks and advantages of hormone replacement } \\
\text { therapy, assessment the needs of minerals (male } \\
\text { sensor } 40+\text { ) }\end{array}$ & ns & & & & \\
\hline & & & $\begin{array}{l}\text { Risk of breast cancer: } 9 \text { gene variations, genetic } \\
\text { checkup program, adjustments in lifestyle, } 13+ \\
\text { gene variations for the effect of } 40 \text { relevant drugs }\end{array}$ & ns & & & & \\
\hline & & & $\begin{array}{l}\text { Primary prevention: } 52 \text { gene variations, more } \\
\text { than } 10 \text { different diseases, effects and adverse } \\
\text { events of } 54 \text { drugs }\end{array}$ & ns & & & & \\
\hline & & & $\begin{array}{l}\text { Gastrointestinal sensor: } 5 \text { gene variations, } \\
2 \text { food intolerances, early detection of Crohn's } \\
\text { disease }\end{array}$ & ns & & & & \\
\hline Geneplanet [40] & Slovenia & $\begin{array}{l}\text { German, } \\
\text { Slovenian, } \\
\text { Croatian, } \\
\text { English, } \\
\text { Hungarian }\end{array}$ & Nutrigenetic analysis & EUR 399 & No & ns & ns & Yes \\
\hline coCap [41] & Germany & $\begin{array}{l}\text { German, } \\
\text { English }\end{array}$ & $\begin{array}{l}\text { Metabolic analysis ( } 3 \text { analyses with different } \\
\text { extension) }\end{array}$ & ca. EUR $330^{6}$ & No & ns & ns & ns \\
\hline \multirow[t]{9}{*}{ Genovia [42] } & \multirow[t]{9}{*}{ Germany } & \multirow{9}{*}{$\begin{array}{l}\text { A variety of } \\
\text { translation } \\
\text { options }\end{array}$} & Hormonal risk of thrombosis during pregnancy & EUR 89 & \multirow[t]{9}{*}{ Yes } & \multirow[t]{9}{*}{ ns } & \multirow[t]{9}{*}{ ns } & \multirow[t]{9}{*}{ Yes } \\
\hline & & & Risk of thrombosis & EUR 89 & & & & \\
\hline & & & Test of drug intolerance & EUR 130 & & & & \\
\hline & & & Test of tamoxifen intolerance & EUR 220 & & & & \\
\hline & & & Macular degeneration & EUR 89 & & & & \\
\hline & & & Lactose intolerance & EUR 89 & & & & \\
\hline & & & Alcohol intolerance & EUR 84.55 & & & & \\
\hline & & & Coffee intolerance & EUR 89 & & & & \\
\hline & & & Obesity and overweight & EUR 84.55 & & & & \\
\hline
\end{tabular}


Table 2 (continued)

\begin{tabular}{|c|c|c|c|c|c|c|c|c|}
\hline \multicolumn{3}{|l|}{ General information } & \multicolumn{3}{|l|}{ Offer } & \multicolumn{3}{|c|}{ Additional information } \\
\hline company & $\begin{array}{l}\text { registered } \\
\text { office }\end{array}$ & languages & health-related tests & costs & $\begin{array}{l}\text { non- } \\
\text { health- } \\
\text { related } \\
\text { tests }\end{array}$ & $\begin{array}{l}\text { sensitivity or } \\
\text { specificity/ } \\
\text { accuracy }\end{array}$ & $\begin{array}{l}\text { reference } \\
\text { to GenDG }\end{array}$ & $\begin{array}{l}\text { certification } \\
\text { and/or } \\
\text { accreditation }\end{array}$ \\
\hline \multirow[t]{5}{*}{ Humatrix [43] } & \multirow[t]{5}{*}{ Germany } & \multirow[t]{5}{*}{ German } & Therapeutic safety of antidepressants & EUR 395.49 & \multirow{5}{*}{ Yes } & \multirow[t]{5}{*}{$\mathrm{Yes}^{4}$} & \multirow[t]{5}{*}{$\mathrm{Yes}^{4}$} & \multirow[t]{5}{*}{ Yes } \\
\hline & & & Therapeutic safety of clopidogrel & EUR 160.88 & & & & \\
\hline & & & Therapeutic safety of statins & EUR 261.43 & & & & \\
\hline & & & Therapeutic safety of contraceptives & ns & & & & \\
\hline & & & Therapeutic safety of tamoxifen & EUR 395.49 & & & & \\
\hline Illid [44] & Germany & German & Metabolic analysis & $\begin{array}{l}\text { EUR } 300- \\
400^{7}\end{array}$ & No & ns & ns & ns \\
\hline Jenagen [45] & Germany & German & Lactose intolerance & EUR 69 & Yes & ns & Yes & Yes \\
\hline \multirow[t]{3}{*}{ LifeCodexx [46] } & \multirow[t]{3}{*}{ Germany } & \multirow{3}{*}{$\begin{array}{l}\text { German, } \\
\text { English, } \\
\text { French, } \\
\text { Italian }\end{array}$} & Trisomy 21 and genetic sex determination & EUR 299 & \multirow[t]{3}{*}{ No } & \multirow[t]{3}{*}{ Yes } & \multirow[t]{3}{*}{ Yes } & \multirow[t]{3}{*}{ Yes } \\
\hline & & & Trisomy $21,18,13$ and genetic sex determination & EUR 349 & & & & \\
\hline & & & $\begin{array}{l}\text { Trisomy } 21,18,13 \text {, test of maldistribution of sex } \\
\text { chromosome, genetic sex determination }\end{array}$ & EUR 399 & & & & \\
\hline \multirow[t]{4}{*}{ Lifegenetics [47] } & \multirow[t]{4}{*}{ Slovenia } & \multirow[t]{4}{*}{ English } & $\begin{array}{l}\text { DNA slim test (nutrition and metabolism and } \\
\text { therefore, weight) }\end{array}$ & EUR 229 & \multirow[t]{4}{*}{ No } & \multirow[t]{4}{*}{ ns } & \multirow[t]{4}{*}{ ns } & \multirow[t]{4}{*}{ ns } \\
\hline & & & $\begin{array}{l}\text { DNA test premium (health and prevention } \\
\text { factors: alcohol metabolism, bone health, caffeine } \\
\text { and nicotine metabolism, cardiovascular health, } \\
\text { celiac disease, detoxification ability of your body; } \\
\text { fat, sugar and insulin regulation, lactose } \\
\text { intolerance, muscular potential and cramps) }\end{array}$ & EUR 279 & & & & \\
\hline & & & DNA test premium + DNA test slim & EUR 299 & & & & \\
\hline & & & $\begin{array}{l}\text { DNA test baby (health and prevention factors: } \\
\text { bone health, caffeine and nicotine metabolism, } \\
\text { cardiovascular health, celiac disease, detoxification } \\
\text { ability of your body; fats, sugar and insulin } \\
\text { regulation, lactose intolerance, muscular potential } \\
\text { and cramps) }\end{array}$ & EUR $279^{8}$ & & & & \\
\hline \multirow[t]{3}{*}{ Meinlabtest [48] } & The & German & Nutrigenetic analysis & EUR 399 & No & ns & ns & Yes \\
\hline & \multirow[t]{2}{*}{ Netherlands } & & $\begin{array}{l}\text { Gene analysis includes } 19 \text { different diseases: } \\
\text { Alzheimer disease, asthma, arterial fibrillation, } \\
\text { basal cell carcinoma, breast cancer, celiac cancer, } \\
\text { colon carcinoma, diabetes type I and II, gallstones, } \\
\text { glaucoma, heart attack, hypertension, lung cancer, } \\
\text { multiple sclerosis, psoriasis, restless leg syndrome, } \\
\text { rheumatoid arthritis, venous thromboembolism) } \\
\text { and analysis of drug reactions (statins, } \\
\text { omeprazole, clopidogrel, metformin, perindopril, } \\
\text { warfarin) as well as analysis of character and } \\
\text { talents (metabolic system, muscle structure, } \\
\text { memory capacities, pain sensitivity, etc.) }\end{array}$ & EUR 499 & & & & \\
\hline & & & Nutrigenetic analysis + personal gene & EUR 799 & & & & \\
\hline \multirow[t]{3}{*}{ MIADNA [49] } & \multirow[t]{3}{*}{ ns } & \multirow[t]{3}{*}{ English } & $\begin{array}{l}\text { Diet and nutrition (exercise potential, eating } \\
\text { behavior, etc.) }\end{array}$ & $\begin{array}{l}\text { USD149 } \\
\text { (EUR } \\
139.79)\end{array}$ & \multirow[t]{3}{*}{ no } & \multirow[t]{3}{*}{ ns } & \multirow[t]{3}{*}{ ns } & \multirow[t]{3}{*}{ ns } \\
\hline & & & $\begin{array}{l}\text { Wellness and lifestyle (hair loss, learning, memory } \\
\text { performance, etc.) }\end{array}$ & $\begin{array}{l}\text { USD } 119 \\
(\text { EUR } \\
111.62)\end{array}$ & & & & \\
\hline & & & $\begin{array}{l}\text { Children's DNA discovery (body height, day or } \\
\text { night person, athletic potential) }\end{array}$ & $\begin{array}{l}\text { USD } 119 \\
(\text { EUR } \\
111.62)\end{array}$ & & & & \\
\hline
\end{tabular}

Health-Related Genetic DTC Tests in the German Setting
Public Health Genomics 2017;20:203-217

DOI: $10.1159 / 000477559$ 
Table 2 (continued)

\begin{tabular}{|c|c|c|c|c|c|c|c|c|}
\hline \multicolumn{3}{|l|}{ General information } & \multicolumn{3}{|l|}{ Offer } & \multicolumn{3}{|c|}{ Additional information } \\
\hline company & $\begin{array}{l}\text { registered } \\
\text { office }\end{array}$ & languages & health-related tests & costs & $\begin{array}{l}\text { non- } \\
\text { health- } \\
\text { related } \\
\text { tests }\end{array}$ & $\begin{array}{l}\text { sensitivity or } \\
\text { specificity/ } \\
\text { accuracy }\end{array}$ & $\begin{array}{l}\text { reference } \\
\text { to GenDG }\end{array}$ & $\begin{array}{l}\text { certification } \\
\text { and/or } \\
\text { accreditation }\end{array}$ \\
\hline \multirow[t]{3}{*}{$\begin{array}{l}\text { Medical Rogaska } \\
{[50]}\end{array}$} & \multirow[t]{3}{*}{ Slovenia } & \multirow[t]{3}{*}{$\begin{array}{l}\text { English, } \\
\text { German, } \\
\text { Russian, } \\
\text { Italian, } \\
\text { Slovenian }\end{array}$} & $\begin{array}{l}\text { Genetic analysis includes } 13 \text { diseases: arterial } \\
\text { fibrillation, stroke, colon and rectum carcinoma, } \\
\text { Crohn's disease, multiple sclerosis, hypertension, } \\
\text { peripheral arterial disease, type } 1 \text { and } 2 \text { diabetes } \\
\text { mellitus, skin cancer, rheumatoid arthritis, } \\
\text { myocardial infarction, chronic kidney disease); for } \\
\text { women additional test of risk of breast and } \\
\text { ovarian cancer, for men additional test of risk of } \\
\text { prostate and lung cancer }\end{array}$ & ns & \multirow[t]{3}{*}{ No } & \multirow[t]{3}{*}{ / } & \multirow[t]{3}{*}{ I } & \multirow[t]{3}{*}{ Yes } \\
\hline & & & $\begin{array}{l}\text { Extended genetic analysis includes } 19 \text { diseases: } \\
\text { additional Alzheimer disease, leukemia, age- } \\
\text { related degeneration, gallstones, asthma, celiac } \\
\text { disease; for women additional test of risk of breast } \\
\text { and ovarian cancer, for men additional test of risk } \\
\text { of prostate and lung cancer }\end{array}$ & ns & & & & \\
\hline & & & $\begin{array}{l}\text { Diet test includes } 35 \text { analyses for body weight, } \\
\text { metabolism and health, vitamins and minerals, } \\
\text { nutrition habits, metabolic efficiency, } \\
\text { detoxification and antioxidants, sport and leisure } \\
\text { time as well as dependencies and ageing; in total } \\
110 \text { gene variations }\end{array}$ & ns & & & & \\
\hline Nifty [51] & China & $\begin{array}{l}\text { Chinese, } \\
\text { Thai, } \\
\text { Romanian, } \\
\text { Korean, } \\
\text { Bulgarian, } \\
\text { Turkish, } \\
\text { Russian, } \\
\text { Polish, } \\
\text { Slovenian, } \\
\text { Arabic, } \\
\text { English }\end{array}$ & $\begin{array}{l}\text { Prenatal test of the most common trisomies } \\
\text { (T21, T18, T22, T16, T9), deletion syndromes, } \\
\text { sex chromosomal aneuploidy }\end{array}$ & $\begin{array}{l}\text { price } \\
\text { information } \\
\text { by physician } \\
\text { or hotline }\end{array}$ & No & Yes & ns & ns \\
\hline Nutrilite [52] & Germany & German & Weight & ns & No & ns & ns & Yes \\
\hline Oncotype [53] & Germany & $\begin{array}{l}\text { German, } \\
\text { English, } \\
\text { French, } \\
\text { Greek, } \\
\text { Spanish, } \\
\text { Portuguese, } \\
\text { Japanese, } \\
\text { Irish }\end{array}$ & Breast cancer test/21 gene expression test & ns & No & ns & ns & ns \\
\hline Prenatalis [54] & Germany & $\begin{array}{l}\text { German, } \\
\text { English }\end{array}$ & Trisomy $21,18,13$ & $\begin{array}{l}\text { EUR 427.94/ } \\
\text { EUR 532.85 }\end{array}$ & & Yes & Yes & Yes \\
\hline & & & Trisomy $21,18,13$, and gonosomal aberrations & $\begin{array}{l}\text { EUR 544.85/ } \\
\text { EUR 649.42 }\end{array}$ & & & & \\
\hline Primahome [55] & Switzerland & $\begin{array}{l}\text { English, } \\
\text { Italian }\end{array}$ & Celiac disease and/or lactose & ns & No & Yes & ns & Yes \\
\hline \multirow[t]{3}{*}{ Progenom [56] } & \multirow[t]{3}{*}{ Germany } & \multirow[t]{3}{*}{ German } & Weight and nutrition & ns & \multirow[t]{3}{*}{ No } & \multirow[t]{3}{*}{ ns } & \multirow[t]{3}{*}{ Yes } & \multirow[t]{3}{*}{ Yes } \\
\hline & & & $\begin{array}{l}\text { Several tests for: breast health sensor; bone health } \\
\text { sensor; toxicological sensor; thrombosis sensor; } \\
\text { cardiovascular sensor; pharmacological sensor; } \\
\text { AMD sensor; glaucoma sensor; diabetes sensor; } \\
\text { hypertension sensor; gluten sensor; lactose sensor; } \\
\text { IBD sensor; Alzheimer sensor; joint sensor; } \\
\text { periodontitis sensor; HIV resistance sensor; iron } \\
\text { sensor; ADHD sensor; female sensor pregnancy; } \\
\text { female health sensor }\end{array}$ & ns & & & & \\
\hline & & & Sport sensor & ns & & & & \\
\hline
\end{tabular}


Table 2 (continued)

\begin{tabular}{|c|c|c|c|c|c|c|c|c|}
\hline \multicolumn{3}{|c|}{ General information } & \multicolumn{3}{|l|}{ Offer } & \multicolumn{3}{|c|}{ Additional information } \\
\hline company & $\begin{array}{l}\text { registered } \\
\text { office }\end{array}$ & languages & health-related tests & costs & $\begin{array}{l}\text { non- } \\
\text { health- } \\
\text { related } \\
\text { tests }\end{array}$ & $\begin{array}{l}\text { sensitivity or } \\
\text { specificity/ } \\
\text { accuracy }\end{array}$ & $\begin{array}{l}\text { reference } \\
\text { to GenDG }\end{array}$ & $\begin{array}{l}\text { certification } \\
\text { and/or } \\
\text { accreditation }\end{array}$ \\
\hline SkinDNA [57] & Germany & German & $\begin{array}{l}\text { Several tests for: accelerate collagen degradation; } \\
\text { reduced protection of glycation; impairment of } \\
\text { UV skin protection; reduced protection for free } \\
\text { radicals; increased risk of inflammation }\end{array}$ & ns & No & ns & ns & ns \\
\hline \multirow[t]{5}{*}{$\begin{array}{l}\text { Whozthedaddy } \\
\text { [58] }\end{array}$} & Canada & English & Diet and nutrition & $\begin{array}{l}\text { GBP 119 } \\
\text { (EUR } \\
138.86 \text { ) } \\
\end{array}$ & Yes & Yes $^{10}$ & ns & Yes \\
\hline & & & Wellness and lifestyle & $\begin{array}{l}\text { GBP 99 } \\
\text { (EUR } \\
115.47 \text { ) }\end{array}$ & & & & \\
\hline & & & Children's DNA discovery & $\begin{array}{l}\text { GBP 99 } \\
\text { (EUR } \\
115.47 \text { ) }\end{array}$ & & & & \\
\hline & & & $\begin{array}{l}\text { Predisposition test includes } 34 \text { different diseases; } \\
\text { immune system: lupus, Graves' disease, celiac } \\
\text { disease, multiple sclerosis, psoriasis; } \\
\text { cardiovascular/cerebrovascular conditions: } \\
\text { intracranial aneurysm, atrial fibrillation, heart } \\
\text { disease, peripheral arterial disease, venous } \\
\text { thromboembolism; aging: Alzheimer disease, } \\
\text { rheumatoid arthritis, osteoporosis; general health: } \\
\text { obesity, migraine, type } 1 \text { and } 2 \text { diabetes, alopecia, } \\
\text { gallstones, sugar consumption, folate metabolism, } \\
\text { vitamin } \mathrm{B}_{6} \text { metabolism, vitamin } \mathrm{B}_{12} \text { metabolism, } \\
\text { vitamin D metabolism; cancer: bladder, breast, } \\
\text { colorectal, gastric, lung, prostate, skin, basal; } \\
\text { ocular (eye) disorders: open-angle glaucoma, } \\
\text { exfoliation glaucoma }\end{array}$ & $\begin{array}{l}\text { GBP 199 } \\
\text { (EUR } \\
232.18) \\
\\
:\end{array}$ & & & & \\
\hline & & & Prenatal testing $(13,18,21, \mathrm{X}, \mathrm{Y})$ & $\begin{array}{l}\text { GBP } 399 \\
\text { (EUR } \\
465.53 \text { ) }\end{array}$ & & & & \\
\hline
\end{tabular}

ns, no information was found. ${ }^{1}$ Cost difference arising due to type of report of result (PDF or book). ${ }^{2}$ The webpage in English is represented by more genetic tests (DNA test dermatological problems etc.). ${ }^{3}$ Advertising with highest accuracy. ${ }^{4}$ Only for paternity. ${ }^{5}$ Different price occurs due to additional individualized receipt book or form of results report. ${ }^{6}$ Price depends on the scope of analysis or service package. ${ }^{7}$ Price depends on consulting service and duration. ${ }^{8}$ Additional shipping costs (EUR 39 or EUR 12). ${ }^{9}$ Price difference results due to duration (5 or $8-10$ working days for transmission of results). ${ }^{10}$ For prenatal testing.

Initially, we wanted to clarify why citizens would like to perform a health-related genetic analysis and which aspects attract them in this market model. In the population, health awareness is a growing issue $[1,63]$. In connection with the ongoing reports of new genetic findings, curiosity and interest about health risks also increase [64, 65]. Vayena [66] investigated the reasons for undergoing genetic testing. However, receiving actionable health information was mentioned as least important, whereas curiosity was stated as the primary reason. This motivation is relieved by an easy access to a genetic analysis through the internet. Thereby, no geographical boundaries and distances from physicians affect the access [14]. Additionally, in most cases, predictive genetic analyses with- out a specific indication were not covered by German statutory health insurances. Hence, genetic analyses are often subject to private payments. For this purpose, genetic analysis via internet seems to be a less expensive alternative to traditional examination by physicians [67].

Generally, the genetic DTC market is an issue in the German setting, and the economic relevance for a solidarily financed health-care system has to be discussed. In the discussion of the potential benefits of health-related genetic analysis, a distinction between lifestyle tests and predictive/diagnostic analysis must be made. Whereas a lower medical value or influence is attributed to lifestyle tests, predictive genetic analyses could have considerable consequences for human health [68]. In recent years, sev- 
eral studies investigated the influence of such health-related genetic analysis on health behavior of the tested person. In the literature, no ambiguous result could be identified. Bloss et al. [69], for example, evaluated the behavior of people after receiving health-related genetic information; no change in behavior with respect to anxiety and diet was determined. In contrast, Kaufman et al. [70] stated that after testing, one-third paid more attention to a healthy diet, $14 \%$ enhanced their physical activity, and $31 \%$ were more determined to exercise. Nonetheless, the strength of the effects on health behavior may depend on the type of analysis. Hence, genetic analysis focusing on lifestyle information has a minor effect on changing behavior than identified genetic mutations in a predictive context. In this context, the psychological burden of patients is a frequently discussed issue [71]. Genetic information could lead to fear of disease, depression, and fear of genetic discrimination $[72,73]$. However, aside from these negative consequences, genetic information may also have a positive impact [74]. A reduction in diseaserelated anxiety may result from the exclusion of a disease, based on genetic analysis [75].

The aforementioned aspects may also have implications in a solidarily financed health-care system and influence the health-care budget. A genetic DTC analysis is performed independently and on the patient's own account. The genetic knowledge gained through the analysis may affect the expenditures on different levels (micro-, meso-, macro-level) of the health-care system. Thereby, patients and physicians are actors on the micro-level, insurances are actors on the meso-level, and the state or the system represents the actors on the macro-level. The effects of health-related genetic testing on the specific levels vary according to the type of the test (predictive/diagnostic or lifestyle). As previously mentioned, lifestyle analyses are attributed a lower impact on health. However, an independent prevention on the basis of the molecular genetic test and lifestyle changes (nutrition and sports) may reduce (private) illness-related subsequent costs for the patients. On the other hand, a lack of success (e.g., weight reduction) may lead to additional/increased use of services of the health-care system. Physicians are faced with more health-conscious and possibly healthier patients, and this may have a positive impact on prescriptive praxis and a number of regulations. An increased number of doctor consultations as well as increased costs of counselling resulting from lifestyle tests may negatively affect physicians on the micro-level. This may lead to an increase in expenses for insurances on the meso-level. However, such kinds of health-related tests may also be ben- eficial. An increase in the use of measures of primary prevention offered by the health insurance (fitness center, nutrition counselling) as well as more health-conscious insured parties may prospectively lead to cost reductions. These cost savings could be achieved by a reduction in subsequent illness-related costs and in the days where one is incapable of work. Overall, considered from the perspective of the system or state, these effects may lead on the one hand to a decrease in expenses for Statutory Health Insurance, and on the other hand to increased human capital caused by a more health-conscious population. Although a negative influence on the expenditure is estimated to be low, in contrast to the discussed lifestyletests, predictive genetic analysis may influence the overall health-care budget significantly. On the micro-level, after receiving predictive test results, positive as well as negative results may occur. The knowledge about the likelihood for one or a variety of specific diseases could induce measures of primary (e.g., precautionary measures, risk reduction) and secondary prevention (e.g., participation in early detection examinations, measures to prevent a manifestation) in the customers. In terms of drug application, genetic test results could lead to a reduction of adverse events. Genetic information may also have a negative effect on patients. This occurs primarily through overestimation of risks [68]. Interested people may become healthy sick people [76], who are characterized by anxiety, uncertainty, and psychological problems. Informed patients may develop other priorities, resulting in an increase of several services and a rise in demand for diagnostic clarifications. Furthermore, family members and, thus, potential risk carriers may become unsettled and increase their demand for medical services. Some of the few positive effects (targeted examination and treatment, close monitoring of risk patients, initiation of precautionary measures, and optimization of medicinal therapy) occur for physicians due to predictive knowledge and some cost-increasing aspects. As a result of uncertainty, patients may increase their demand for doctor consultations and also increase the expense of counselling. Due to the predictive findings, an additional or renewed diagnostic clarification is often necessary. Basically, predictive findings may encourage a demand-induced supply. Effects of the micro-level influence the effects as well the cost-situation for insurances. Early intervention options for risk patients may reduce follow-up costs in terms of avoiding disease manifestations, timely prevention, treatment, and reduce days where one is unable to work. Negative effects may result in costs for additional (partly unnecessary) medical services (e.g., costs to verify 
results). Further issues in this context for insurances are indirect risk selection, migration of good risks to private insurance companies, increase of expenses, and thus an increase of additional contributions. Overall, this means a softening of the term "indication" for the system, which may lead to a service extension. The oversupply or misuse of results may increase expenses in a solidarily financed health-care system. However, on the other hand, predictive results may also have positive effects on the healthcare budget as well as lead to improved cost-effectiveness of medical measures through targeted use.

In summary, positive and negative effects may occur due to genetic DTC tests for a solidarily financed healthcare system. At the moment, a clear statement regarding the effects that will dominate can hardly be assessed. In a system with many chronic diseases, behavior changes or a more frequent use of medical check-ups may be beneficial [77]. However, this presumes that customers understand this genetic knowledge and are able to deal with deterministic results $[78,79]$. German providers of predictive and diagnostic DTC tests have to ensure the integration of a physician and/or a genetic counselling for such analysis. Advised customers are more informed and are better able to deal with probabilistic results. Hence, the strength of the negative effects is counteracted by comprehensive explanation and counselling. As previously explained, however, access is not limited to offers of specific countries or of a certified quality. People can purchase genetic DTC tests without integrating a physician, and this may particularly lead to predictive analysis, which has negative consequences for the players on all levels. Therefore, it is the task of the government to develop information structures for the consolidation of public consciousness and consumer sovereignty (information about advantages and disadvantages, chances and risks, quality criteria of analysis, etc.), and expanding infrastructure for human genetic counselling. Further, insurances have the possibility of informing their insured community through awareness campaigns or offers about the chances and risks of genetic analysis, especially regarding genetic DTC tests.

This systematic review has some limitations. In the search strategy, only German terms were used to evaluate the offers, which focus on the German population. However, using English terms for searching genetic analyses may increase the number of hits significantly. However, search terms such as "DNA test" and "DNS test" are the same in German and English. Hence, these lead to an inclusion of webpages, which are providing only information in English. Moreover, this affects only $11 \%$ of the

Health-Related Genetic DTC Tests in the German Setting included webpages. The internet constitutes a possibility to get access to the global market and, therefore, interested customers may choose from a multitude of offers. This underlines the need of a comprehensive information and education about the risks and concerns of genetic DTC tests. A sole search with language-specific terms leads, despite a highly restricted market, to a plurality of international offers. This may lead to risks or negative consequences for an uninformed citizen who relies on national regulations. Therefore, the population should also be made aware of international offers with possible questionable values and the importance of genetic counselling. Furthermore, using another search engine (e.g., Bing or Yahoo) may lead to other results. However, Google has the highest market share in Germany [80]; thereby, this analysis shows the representative spectrum of offers a German consumer will receive if she or he is searching for genetic tests on the internet. Furthermore, it could be assumed that companies oriented their search engine optimization with Google for a better retrievability. At least, a further limitation could be seen in the potential implications for a solidarily financed health-care system. These implications are speculative as well as not supported by any data. At the time of our study, we did not identify specific economic literature or data for the monetary benefits and/or consequences of genetic DTC testing. However, this discussion may increase the awareness in the different player and policy decision makers of potential cost-increasing consequences as well as lead to further discussions.

\section{Conclusion}

The progress in genomics has led to an increased offer of genetic analysis. In Germany, the execution of healthrelated genetic DTC tests, especially for predictive or diagnostic purposes, is restricted by the GenDG. However, the globalization enables a worldwide access to genetic DTC tests via the internet. Hence, also people in a highly regulated market are confronted with a majority of international offers. This global genetic DTC market is characterized by lack of transparency, and for the individual these various offers are partly or completely not comparable. Primarily, genetic DTC tests are performed on people's own account, but may lead to subsequent costs for a solidarily financed health-care system. Therefore, information and education about chances and risks of genetic DTC tests is a future responsibility of policy decision makers. 


\section{Disclosure Statement}

The authors declare no conflicting interests.

\section{Author Contributions}

Marika Plöthner, the lead author, created the study design and initial draft. Mike Klora, Daniel Rudolph, and Johann-Matthias Graf von der Schulenburg contributed to the manuscript through literature search, discussions on design and structure, writing, and reviews.

\section{References}

1 Tulchinsky TH, Varavikova E: The New Public Health, ed 2. Amsterdam/Boston, Elsevier/ Academic Press, 2009.

2 Berg S: The well-informed patient: a new breed of health care consumer. Asthma Mag 2005; 10:28-30

3 Diaz JA, Griffith RA, Ng JJ, Reinert SE, Friedmann PD, Moulton AW: Patients' use of the Internet for medical information. J Gen Intern Med 2002;17:180-185.

4 Tonsaker T, Bartlett G, Trpkov C: Health information on the Internet: gold mine or minefield? Can Fam Physician 2014;60:407-408.

5 Hübner M: Dr. Google setzt Ärzte unter Druck. Ärztezeitung online. 2016. http:// www.aerztezeitung.de/praxis_wirtschaft/ special-arzt-patient/article/913353/umfragezeigt-dr-google-setzt-aerzte-druck.html (accessed October 6, 2016).

6 National Institutes of Health: Biological Sciences Curriculum Study. Understanding Human Genetic Variation. 2007. https://www. ncbi.nlm.nih.gov/books/NBK20363/ (accessed December 6, 2016).

7 Hayden EC: Technology: The $\$ 1,000$ genome. Nature 2014;507:294-295.

8 Manolio TA, Collins FS: The HapMap and genome-wide association studies in diagnosis and therapy. Annu Rev Med 2009;60:443456.

9 National Human Genome Research Institute: Genome-Wide Association Studies. 2015. https://www.genome.gov/20019523/ (accessed December 6, 2016).

10 Rockman MV: Reverse engineering the genotype-phenotype map with natural genetic variation. Nature 2008;456:738-744.

11 Wray NR, Goddard ME, Visscher PM: Prediction of individual genetic risk to disease from genome-wide association studies. Genome Res 2007;17:1520-1528.

12 O'Daniel JM: The prospect of genome-guided preventive medicine: a need and opportunity for genetic counselors. J Genet Counsel 2010; 19:315-327.

13 Mihaescu R, Moonesinghe R, Khoury MJ, Janssens AC: Predictive genetic testing for the identification of high-risk groups: a simulation study on the impact of predictive ability. Genome Med 2011;3:51.

14 Su P: Direct-to-consumer genetic testing: a comprehensive view. Yale J Biol Med 2013;86: 359-365.
15 Liu Y, Pearson YE: Direct-to-consumer marketing of predictive medical genetic tests: assessment of current practices and policy recommendations. J Public Policy Market 2008; 27:131-148.

16 Global Industry Analysts: Future of Directto-Consumer (DTC) Genetic Testing Market Remains Fraught. 2012. http://www.prweb. com/releases/DTC_genetic_testing/direct_ to_consumer_tests/prweb9780295.htm (accessed December 6, 2016)

17 US National Library of Medicine: What is direct-to-consumer genetic testing? 2016 https://ghr.nlm.nih.gov/primer/testing/directtoconsumer (accessed December 7, 2016).

18 Lippi G, Favaloro EJ, Plebani M: Direct-toconsumer testing: more risks than opportunities. Int J Clin Pract 2011;65:1221-1229.

19 Kalokairinou L, Howard HC, Borry P: Directto-Consumer Genetic Testing. Chichester, John Wiley and Sons, 2001.

20 The German Reference Centre for Ethics in the Life Sciences (DRZE): Predictive Genetic Testing-Medical and scientific aspects. 2011 http://www.drze.de/in-focus/predictive-genetic-testing? set_language $=$ en $($ accessed December 7, 2016).

21 Gollust SE, Wilfond BS, Hull SC: Direct-toconsumer sales of genetic services on the Internet. Genet Med 2003;5:332-337.

22 Global Industry Analysts: Genetic testing - a global strategic business report. 2015. http:// www.strategyr.com/pressMCP-1392.asp (accessed December 12, 2016).

23 XE Currency Converter. Exchange Rates. 2016. http://www.xe.com/ (accessed December 2, 2016).

24 Abnehmgene: Diverse webpage information. 2015. www.abnehmgene.com (accessed November 17, 2016).

25 Adisonlab: Diverse webpage information 2014. http://www.adisonlab.com/ (accessed November 17, 2016)

26 Amway: Diverse webpage information. 2016. www.amway.de (accessed November 17, 2016).

27 Ceneta: Diverse webpage information. 2016. www.cenata.de (accessed November 17, 2016).

28 Coloalert: Diverse webpage information. 2015. http://www.coloalert.de/ (accessed November 17, 2016).
29 DNAdirekt: Diverse webpage information. 2016. http://www.dna-direkt.de/ (accessed November 17, 2016).

30 DNAfit: Diverse webpage information. 2016. https://www.dnafit.com (accessed November 17, 2016).

31 DNA Plus-Zentrum für Humangenetik: diverse webpage information. 2009. http:// www.dnaplus.de/ (accessed November 17, 2016).

32 DTC DNA Testing Center: Diverse webpage information. 2008. http://dnatestingcenter. de/ (accessed November 17, 2016).

33 DNAnutriControl: Diverse webpage information. 2014. http://www.dnanutricontrol. com/ (accessed November 18, 2016).

34 DNA Weight Control: Diverse webpage information. 2014. http://www.dnaweightcontrol.ch/ (accessed November 17, 2016).

35 Dr. Seibt Genomics: Diverse webpage information. 2016. www.dr-seibt-genomics.com (accessed November 17, 2016).

36 EasyDNA: Diverse webpage information. 2016. http://www.easydna.ch/ (accessed November 17, 2016)

37 FutureGenetic: Diverse webpage information. 2016. https://future-genetik.com/ (accessed November 17, 2016).

38 Genetic balance: Diverse webpage information. 2016. https://genetic-balance.com (accessed November 17, 2016).

39 Genoris: Diverse webpage information. 2015. https://genoris.com (accessed November 17, 2016).

40 Geneplanet: Diverse webpage information. 2016. www.geneplanet.de (accessed November 17, 2016).

41 coCap: Diverse webpage information. 2016. www.cogap.de (accessed November 17, 2016).

42 Genovia: Diverse webpage information. 2012. http://genovia-shop.de/ (accessed November 17, 2016).

43 Humatrix: Diverse webpage information. 2016. www.humatrix.de (accessed November 17, 2016)

44 Ilid: Diverse webpage information. 2016. http://www.llid-metacheck.de/ (accessed November 18,2016$)$

45 Jenagen: Diverse webpage information. 2016. http://www.jenagen.de/ (accessed November $18,2016)$ 
46 Lifecodexx: Diverse webpage information. 2015. http://lifecodexx.com/ (accessed November 18, 2016).

47 Lifegenetics: Diverse webpage information. 2016. http://lifegenetics.net/ (accessed November 18,2016 ).

48 Meinlabtest: Diverse webpage information. 2016. http://www.meinlabtest.de/ (accessed November 18, 2016).

49 MIADNA: Diverse webpage information. 2016. http://www.miadna.com/ (accessed November 18, 2016)

50 Medical Rogaska: Diverse webpage information. 2015. http://www.rogaska-medical. com/ (accessed November 18, 2016).

51 Nifty: Diverse webpage information. 2014. www.niftytest.com (accessed November 18, 2016).

52 Nutrilite: Diverse webpage information. 2016. https://nutrilitebodykey.de (accessed November 18, 2016).

53 Oncotype: Diverse webpage information. 2016. http://www.oncotypedx.com/ (accessed November 18, 2016).

54 Prenatalis: Diverse webpage information. 2016. www.prenatalis.de (accessed November 18, 2016).

55 Primahome: Diverse webpage information. 2016. https://primahometest.com/ (accessed November 18, 2016).

56 Progenom: Diverse webpage information. 2016. http://www.progenom.com/ (accessed November 18, 2016).

57 SkinDNA: Diverse webpage information. 2015. www.skindna.de (accessed November $18,2016)$.

58 Whózthedaddy: Diverse webpage information. 2016. https://www.whozthedad-dy.com (accessed November 18, 2016).

59 Borry P, van Hellemondt RE, Sprumont D, et al: Legislation on direct-to-consumer genetic testing in seven European countries, Eur J Hum Genet 2012;20:715-721.

60 EASAC-FEAM Working Group on DirectTo-Consumer Genetic Testing: Announcement of new project and open Call for Evidence. 2011. http://www.easac.eu/biosciences/current-projects/direct-to-consumergenetic-testing.html (accessed May 5, 2016).
61 Human Genetics Commission: A Common Framework of Principles for Direct-to-Consumer Genetic Testing Services. 2010. http:// www.hgc.gov.uk/Client/document. asp? DocId $=280 \&$ CAtegoryId $=10$ (accessed May 5, 2016).

62 Lek M, Karczewski KJ, Minikel EV, Samocha KE, Banks E, Fennell T, et al: Analysis of protein-coding genetic variation in $60,706 \mathrm{hu}-$ mans. Nature 2016;536:285-291.

63 Business Wire: Research and Markets: Global Health and Wellness Food Market 2015-2019 - Growing Health Awareness among Consumers. 2016. http://www.businesswire.com/ news/home/20160111005643/en/ResearchMarkets-Global-Health-Wellness-FoodMarket (accessed December 12, 2016).

64 Gollust SE, Gordon ES, Zayac C, Griffin G, Christman MF, Pyeritz RE, et al: Motivations and perceptions of early adopters of personalized genomics: perspectives from research participants. Public Health Genomics 2012; 15:22-30.

65 Anderson AE, Flores KG, Boonyasiriwat W, Gammon A, Kohlmann W, Birmingham WC, et al: Interest and informational preferences regarding genomic testing for modest increases in colorectal cancer risk. Public Health Genomics 2014;17:48-60.

66 Vayena E, Gourna E, Streuli J, Hafen E, Prainsack B: Experiences of early users of direct-toconsumer genomics in Switzerland: an exploratory study. Public health Genomics 2012;15:352-362.

67 Altman RB: Direct-to-consumer genetic testing: failure is not an option. Clin Pharmacol Ther 2009;86:15-17.

68 Roberts JS, Ostergren J: Direct-to-consumer genetic testing and personal genomics services: a review of recent empirical studies. Curr Genet Med Rep 2013;1:182-200.

69 Bloss CS, Schork NJ, Topol EJ: Effect of direct-to-consumer genomewide profiling to assess disease risk. N Engl J Med 2011;364: 524-534.

70 Kaufman DJ, Bollinger JM, Dvoskin RL, Scott JA: Risky business: risk perception and the use of medical services among customers of DTC personal genetic testing. J Genet Counsel 2012;21:413-22.
71 Bloss CS, Wineinger NE, Darst BF, Schork NJ, Topol EJ: Impact of direct-to-consumer genomic testing at long term follow-up. J Med Genet 2013;50:393-400.

72 National Human Genome Research Institute: Genetic Discrimination. 2016._https://www. genome.gov/10002077/genetic-discrimination/ (accessed December 7, 2016).

73 Patenaude AF: Addressing Psychological Impacts of Genetic Testing on Patients, Families. Washington, American Psychological Association, 2013. http://www.apa.org/news/ press/releases/2013/05/genetic-testing.aspx (accessed December 7, 2016).

74 O’Neill SC, Tercyak KP, Baytop C, Hensley Alford S, McBride CM: A new approach to assessing affect and the emotional implications of personal genomic testing for common disease risk. Public Health Genomics 2015; 18:104-112.

75 Hilgart JS, Coles B, Iredale R: Cancer genetic risk assessment for individuals at risk of familial breast cancer. Cochrane Database Syst Rev 2012;CD003721.

76 Hubbard R: Predictive genetics and the construction of the healthy ill. Suffolk Univ Law Rev 1993;27:1209-1224.

77 Jamison DT: Disease Control Priorities in Developing Countries, ed 2. New York, Washington, Oxford University Press, 2006.

78 McPherson E: Genetic diagnosis and testing in clinical practice. Clin Med Res 2006;4:123129.

79 Ostergren JE, Gornick MC, Carere DA, Kalia SS, Uhlmann WR, Ruffin MT, et al: How well do customers of direct-to-consumer personal genomic testing services comprehend genetic test results? Findings from the Impact of Personal Genomics Study. Public Health Genomics 2015;18:216-224.

80 Statista: Market share of leading search engines in Germany between 2014 and 2016. 2017. https://de.statista.com/statistik/daten/ studie/167841/umfrage/marktanteile-ausgewaehlter-suchmaschinen-in-deutschland/ (accessed May 2, 2017).
Health-Related Genetic DTC Tests in the German Setting
Public Health Genomics 2017;20:203-217 DOI: $10.1159 / 000477559$ 